\title{
Модели оценки способностей управляющих активами к микро- и макропрогнозированию
}

\author{
Семушин А.В. ${ }^{26}$, Паршаков П.А. ${ }^{27}$
}

Вложение средств в паи инвестиционных фондов является одним из наиболее популярных инструментов, используемых населением для сбережения. Данная работа предлагает обзор и критику основных подходов к оченке способностей управлящих ПИФами $\kappa$ макропрогнозированию (маркет-таймингу) и микропрогнозированию (пикинг, селектинг).

JEL: G11, G23

Ключевые слова на русском: ПИФ, пикинг, тайминг, способности, навык

\section{1. Введение}

При анализе эффективности активного управления выделяют два основных фактора: способности к макропрогнозированию и микропрогнозированию. Первое предполагает, что менеджер обладает необходимыми навыками, которые позволяют ему угадывать движения всего рынка в целом, то есть заниматься маркет-таймингом. Микропрогнозирование же заключается в поиске недооцененных активов, приобретение которых способно принести прибыль в будущем. Изначально подобное разделение было предложено Юджином Фамэ, который в 1972 году параллельно с Майклом Дженсеном пришел к выводу, что ранее предложенные меры могут давать неадекватные результаты при замерах эффективности на практике, так как они не позволяют достаточно точно оценить влияние различных составляющих процесса управления при нестационарности риска портфеля (Fama, 1972). Несколько позже Блэк и Трейнор сумели обосновать вывод, согласно которому менеджеры способны эффективно отделять свои решения в области выбора активов от выбора времени инвестирования (Treynor and Black, 1973). Более того, дальнейшие исследования также подтвердили, что портфели взаимных фондов не могут быть охарактеризованы постоянным уровнем риска, что объясняется изменяющимся влиянием разных составляющих процесса принятия решения об инвестировании на итоговый результат (Stanley and Frank, 1978).

\section{2. Анализ способностей управляющих к выбору актива}

Как уже отмечалось, под способностями к пикингу (piking) понимается умение управляющего находить среди множества всех доступных альтернатив активы, которые на момент анализа являются недооцененными рынком и ожидаемая доходность по которым в будущем будет выше ожидаемой доходности по рынку. С некоторой степенью уверенности можно сказать, что этот навык является близким к фундаментальному анализу ценных бумаг.

\section{1. Безусловные меры}

Одной из наиболее популярных мер, которая может быть использована для оценки способностей к выбору активов, является альфа Дженсена, которая была предложена им в статье 1969 года (Jensen, 1972b). Существует большое количество мер, логика построения которых аналогична ей. Расчет данного показателя определяется следующей формулой:

\footnotetext{
${ }^{26}$ Аспирант Пермского государственного национального исследовательского университета.

27 Преподаватель кафедры оценки стоимости активов Национального исследовательского университета «Высшая школа экономики» (Пермь).
} 


$$
\alpha=\left(\bar{r}-\overline{r_{f}}\right)-\beta_{p}\left(\overline{r_{m}}-\overline{r_{f}}\right)
$$

где $\bar{r}_{m}$ - доходность рыночного портфеля, ${ }^{-}$- доходность по портфелю фонда. Стоит отметить, что основная идея, использованная для вывода данного показателя является достаточно простой. Перегруппируем слагаемые в правой части вышеописанного равенства, чтобы получить следующий результат:

$$
\alpha=\bar{r}-\left(\overline{r_{f}}+\beta_{p}\left(\overline{r_{m}}-\overline{r_{f}}\right)\right) .
$$

Таким образом, выражение, стоящее в общих скобках, представляет собой не что иное, как ожидаемую доходность портфеля фонда согласно модели ценообразования на капитальные активы (САРM). Следовательно, сама альфа описывает то, какая часть фактической доходности фонда была создана особыми способностями управляющего, которые позволили ему превзойти рынок. Бета же, согласно логике построения модели, является постоянной, вследствие чего можно утверждать, что признаком наличия у управляющего способностей к выбору актива является его умение принимать на себя недиверсифицируемую составляющую риска и при этом получать за это дополнительную доходность выше рыночной.

Несложно догадаться, что положительное значение альфы будет свидетельствовать о наличии отличительных способностей, и наоборот. Тем не менее хотелось бы отметить, что, несмотря на кажущуюся простоту рассматриваемого показателя, его автор сделал достаточно серьезный шаг в развитии оценки навыков управляющих: Дженсен одним из первых использовал в качестве бенчмарка не просто безрисковую доходность, а попытался добиться согласованности уровня риска стратегии фонда и эталонного портфеля.

Рассмотрим действия управляющего некоторого условного фонда, который обладает способностями как к выбору ценных бумаг, так и к выбору времени инвестирования. Можно предположить, что в каждый момент времени доходность его стратегии будет определяться тем, какие решения были приняты им в обеих областях. При этом бета будет являться показателем присутствия фонда в рыночной позиции, а альфа станет характеризовать избыточную доходность, которая будет получена за счет отбора ценных бумаг. Тем не менее при практических расчетах определение альфы Дженсена может потребовать построения линейной регрессии аналогично мере Трейнора. Соответственно, мы столкнемся с тем, что бета будет усредняться и фиксироваться на постоянном уровне, что будет приводить к искажениям, в том числе и оценок альфы. Для того чтобы избежать подобных казусов, мы вынуждены изначально предполагать, что управляющий рассматриваемого фондов неспособен правильно выбрать время инвестиций. То есть, располагая только данными о прежних доходностях фонда, невозможно разделить для последующей оценки способности его управляющего в области пикинга и тайминга. Вследствие чего необходимо введение допущения о неспособности управляющего к таймингу (Jensen, 1972b).

Отдельно стоит отметить, что в логике построения альфы Дженсена существует весомый недостаток, заключающийся в следующем: выбор бумаги, ожидаемая доходность по которой выше рыночной и увеличение ее веса в портфеле приводит к тому, что меняется и диверсифицируемая составляющая риска, отличная от заложенной в САРМ, что искажает оценки альфы. Во избежание этого Трейнор и Блек предложили собственную корректировку получаемых оценок путем нормирования по данному отклонению (Treynor, 1973):

$$
\alpha_{\text {corr }}=\frac{\alpha}{\sigma_{e}},
$$
альфы.

где $\sigma_{e}$ - стандартное отклонение остатков регрессии, полученной в процессе оценки Модель САРМ не является единственной используемой для описания ценообразования 
на активы на финансовых рынках. В результате этого альфа Дженсена претерпела множество модификаций, основной смысл которых, как правило, сводится к замене доходности по бенчмарку на предсказываемую той или иной моделью.

В качестве примера подобной модификации можно привести трехфакторную альфу, разработанную Фамэ и Френчем. В своей статье 1992 года они отмечали, что САРМ недостаточно точно описывает цены, складывающиеся на рынке, а добавление к регрессии двух дополнительных факторов значительно улучшает результаты. В итоге в 1993 году ими была предложена следующая формула оценки способностей к выбору активов (Fama and French, 1993):

$$
\alpha=\left(\bar{r}-\bar{r}_{f}\right)-\beta_{1}\left(\bar{r}_{m}-\bar{r}_{f}\right)-\beta_{2} S M B-\beta_{3} H M L .
$$

В данном случае SMB представляет собой так называемый фактор размера, который определяется как разница в доходности по акциям крупных и малых компаний. Фамэ и Френч предлагали использовать в качестве признака размера объем капитализации. В итоге для расчета фактора размера, компании, акции которых торгуются на рынке, разбиваются на две группы согласно их размеру, после чего доходности по группам усредняются и их разница принимается равной значению искомого показателя. Аналогичным же образом происходит и определение переменной $\mathrm{HML}$, с учетом замены используемого классификационного признака на мультипликатор P/BV.

Схожим образом построил свою меру и Кархарт, предложивший дополнительно ко всему учитывать и фактор моментума (Carhart, 1997):

$$
\alpha=\left(\bar{r}-\bar{r}_{f}\right)-\beta_{1}\left(\overline{r_{m}}-\bar{r}_{f}\right)-\beta_{2} S M B-\beta_{3} H M L-\beta_{4} W M L
$$

WML при этом представляет собой разницу в результатах лучших и худших активов. Для ее построения из всего множества доступных альтернатив выбираются бумаги с наиболее высокой и наиболее низкой доходностью за прошедший период, после чего величина разброса экономической отдачи между ними принимается равной WML.

В целом логика построения подобных мер в некотором смысле совпадает с предложенной ранее Уильямом Шарпом методикой для определения инвестиционного стиля управляющих (Martin, 2007). При этом оценка коэффициента бета перед каждым из факторов будет характеризовать то, какую роль каждый актив играет в формировании результата фонда. Если более внимательно рассмотреть показатель Кархарта, то можно отметить, что в данном случае доходность по бенчмарку представляет собой суррогатный портфель, в который с разными весами включаются рыночный портфель, торговая стратегия, эксплуатирующая аномалию, согласно которой малые компании показывают более высокий уровень доходности, нежели большие; стратегию, основывающуюся на выборе между акциями роста и акциями стоимости; а также стратегию выбора между сохранением относительной доходности активов на рынке и ее падением.

\section{2. Условные меры}

Описанные выше меры способностей к выбору актива представляют собой безусловные альфы, которые не учитывают возможность того, что менеджер фонда будет изменять состав портфеля (и его риск), основываясь на различной во времени информации об ожидаемой доходности и риске отдельных активов. В результате этого беты могут оставаться непостоянными во времени, что, аналогично наличию способностей к выбору времени инвестирования, может внести искажения в оценки альфы. В некоторой степени данного недостатка можно избежать путем использования условных мер, которые ниже будут рассмотрены нами более подробно.

Стоит отметить, что все вышеприведенные показатели могут быть определены как с помощью регрессионного анализа, так и стандартных логико-алгебраических методов. Разница при этом заключается в том, что в первом случае (то есть при параметрическом 
подходе к атрибуционному анализу эффективности) на основании данных строится регрессия, которая, как правило, представляет собой модель с распределенными лагами, а оценки ее параметров принимаются в качестве значений мер способностей управляющего. Подобное решение требует использования достаточно большого количества наблюдений в целях достижения статистической значимости получаемых выводов (в рамках асимптотического подхода). Более того, на практике оно часто может быть сопряжено с проблемами, вызванными нестационарностью процессов генерации временных рядов используемых данных. Второй же подход является непараметрическим и предполагает отдельное определение значения меры для каждого временного промежутка в выборке, после чего полученная совокупность результатов подвергается анализу с помощью статистических методов.

\section{3. Анализ способностей управляющих к таймингу}

По аналогии с тем, как нами определялись способности управляющих к выбору активов, можно сказать, что под навыками выбора времени входа в рынок понимается умение производить диверсификацию управляемого портфеля таким образом, чтобы она соответствовала инвестиционным ориентирам собственников паев. То есть маркет-тайминг представляет собой не что иное, как управление систематическим риском вложений.

Стоит понимать, что под таймингом может пониматься не только вход на один конкретный рынок. В частности, Глассман и Ридик исследовали результаты деятельности американских взаимных фондов в конце 1980-х - начале 1990-х годов на предмет наличия у них способностей к таймингу мирового рынка (движение ресурсов между всеми рынками капитала) и национального рынка (совершение операций только на американском рынке) (Glassman and Riddick, 2003). При этом существование второго типа способностей было доказано, в отличие от первого.

В целом все подходы к оценке способностей к выбору времени инвестирования можно разделить на две основные группы: подходы, основанные на анализе рядов доходностей фондов, и подходы, основанные на анализе состава портфеля. Последние предполагают рассмотрение структуры портфеля на предмет того, насколько эффективно менеджер распределяет средства, чтобы избежать просадки капитала при падении рынка или его увеличения при росте. Тем не менее их использование сильно ограничено по причине закрытости информации, требующейся для подобного исследования. Дело в том, что фонды, как правило, предоставляют инвесторам данные о структуре портфеля только в своих квартальных отчетах. Если принять во внимание тот факт, что история подобных наблюдений является достаточно недолгой, то становится очевидным, что оценки, которые могут быть получены при этом, вряд ли окажутся справедливыми. Более того, некоторые управляющие намеренно искажают информацию о составе портфеля, заполняя позиции в определенных бумагах непосредственно перед отчетной датой. В некоторой степени эти ограничения попытались обойти Грэхем и Харви, рассматривавшие динамику структуры искусственных портфелей, операции по которым совершались в соответствии с ежедневными рекомендациями экспертов в газетах (Graham and Harvey, 1996).

Большую популярность завоевали подходы к оценке навыка поиска времени входа в позицию, основанные на анализе исторических рядов доходностей. Логично предположить, что если мы все же рассматриваем диверсифицируемую часть риска, то, в соответствии с современными моделями ценообразования, именно бета может быть использована для оценки способностей менеджера к таймингу рынка. Во всех вышеприведенных моделях альф, используемых для оценки навыков выбора активов, бета теоретически также может отражать тайминг способности. Тем не менее на практике существование у управляющего одновременно двух групп навыков приводит к тому, что оценки параметров регрессии искажаются, так как тайминг и пикинг не могут быть эффективно разделены в рамках обобщенных (не атрибуционных) моделей оценки. Более того, представленные выше меры 
предполагают, что бета портфеля остается постоянной на ее среднем уровне, и это приемлемо при оценке способностей управляющих к выбору актива, но неприемлемо, когда внимание концентрируется непосредственно на навыках к предсказанию движения рынка.

Одними из первых исследователей, разработавших тест на тайминг, с учетом возможности изменения беты были Трейнор и Мазуи (Treynor and Mazuy, 1966). Предложенный подход предполагал, что менеджеры постоянно пытаются переиграть рынок и при этом действуют в рамках области между высокой волатильностью рынка и низкой волатильностью. Таким образом, при решении задачи максимизации результата за счет выбора времени совершения операции менеджер будет увеличивать бету при ожидании положительной рыночной доходности, и снижать ее в обратном случае. Именно на этой достаточно простой идее и основана большая часть тестов на маркет-тайминг. Тем не менее нет оснований считать, что фонд будет идеально предсказывать динамику рынка, поэтому Трейнор и Мазуи полагали, что зависимость доходности фонда от рыночной доходности при наличии навыков к таймингу будет иметь квадратичную, а не линейную форму. В результате предложенная модель может быть записана в виде:

$$
r_{p, t+1}=\alpha_{p}+\beta_{p} r_{m, t+1}+\gamma_{p}\left(r_{m, t+1}\right)^{2}+e_{p, t+1}
$$

где ${ }^{\gamma_{p}}$ - мера особых навыков менеджера к таймингу, а $e_{p, t+1}$ - случайный остаток. При этом стоит заметить, что альфа будет отражать вознаграждение за недиверсифицируемый риск и характеризовать способности к выбору активов.

Однако необходимо отметить, что вышеописанная спецификация будет являться справедливой только при том условии, что управляющий изменяет бету портфеля в зависимости от прогноза достаточно плавно. В частности, Адмати с соавторами расширили предложенную модель и для того, чтобы обеспечить выполнение данного условия, описали функцию, согласно которой менеджер будет изменять бету в зависимости от полученного им сигнала (Admati, Bhattacharya, Ross and Pfleiderer, 1986). Они предполагали, что если управляющий, действующий и при этом абсолютно не склонный к риску, получает зашумленный сигнал о будущей рыночной доходности, то он будет определять бету портфеля в соответствии со следующей формулой:

$$
\beta_{t}=\bar{\beta}+\lambda\left(r_{m, t+1}-E\left(r_{m}\right)+\eta\right),
$$

где $\bar{\beta}$ - сложившийся на момент принятия решения уровень беты, $\lambda-$ мера агрессивности реакции менеджера на полученный сигнал, $\eta$ - нормально распределенный шум.

Дальнейшее развитие данный подход получил в 1996 году, когда Ферсон и Шадт предложили модель с условной бетой, в основе которой лежало расширение стандартной CAPM, предполагающее изменение беты портфеля в зависимости от множества общедоступной информации (Ferson and Schadt, 1996). При этом условная бета может быть описана следующим образом для информационного множества $\mathrm{Z}_{\mathrm{t}}$ :

$$
\beta_{i}=\beta_{0}+B_{i}^{\prime}\left(z_{t}\right)
$$

где ${ }^{z_{t}}$ - отклонение $\mathrm{Z}_{\mathrm{t}}$ от безусловных мер, $\beta_{0}$ - средняя безусловная бета.

Несколько позже данные выводы были использованы и применительно к альфе, в результате чего условная альфа была определена следующим образом (Christopherson,Ferson and Glassman, 1998:

$$
\alpha_{i}=\alpha_{0}+A_{i}^{\prime}\left(z_{t}\right)
$$

где $\alpha_{0}$ - средняя безусловная альфа.

В результате условная САРМ имеет форму:

$$
r_{i, t+1}=\alpha_{i}+A_{i}^{\prime}\left(z_{t}\right)+\beta_{0}\left(r_{\text {bench }, t+1}\right)+B_{i}^{\prime}\left(z_{t}^{*} r_{\text {bench }, t+1}\right)+e_{i, t+1},
$$


где $r_{\text {bench }, t+1}$ - доходность по бенчмарку в периоде $t+1$.

Достаточно интересным является тот факт, что данное расширение может быть использовано для всех ранее рассмотренных нами параметрических тестов, предназначенных для оценки пикинг- и тайминг-способностей. Исследования Ферсона и Шадта, а также Кристоферсона, Глассмана и Ферсона показали, что применение условных мер приводит к росту предсказательной силы моделей, используемых для оценки эффективности управляющих, что во многом объясняется тем фактом, что подобный подход позволяет учесть возможность изменения риска портфеля фонда (Mandelker and Rhee, 1984).

Возвращаясь к мере Трейнора и Мазуи, предположим, что векторы $z_{t}$ и

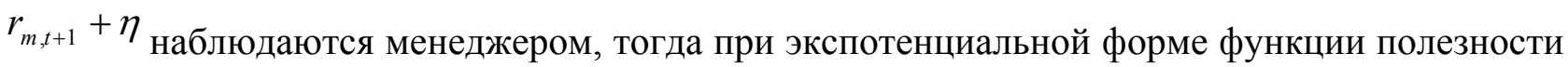
управляющего его спрос на рисковый актив, роль которого при маркет-тайминге играет рыночный портфель, будет характеризоваться линейной зависимостью от информации. Так как при постановке задачи тайминга место имеют лишь два актива, один из которых является безрисковым, то бета, определяющая долю рискового актива в портфеле фонда, будет являться линейной функцией от векторов $z_{t}$ и $r_{m, t+1}+\eta$. Таким образом, модель Трейнора и Мазуи с условной бетой может быть записана следующим образом:

$$
r_{p, t+1}=\alpha_{p}+\beta_{p} r_{m, t+1}+C_{p}^{\prime}\left(z_{t} r_{m, t+1}\right)+\gamma_{p}\left(r_{m, t+1}\right)^{2}+e_{r, t+1},
$$

где $C_{p}$ представляет собой меру реакции беты портфеля на изменения во множестве общедоступной информации. Более того, при подобном подходе $\gamma_{p}$ будет описывать чувствительность беты к частному сигналу о движении рынка в будущем. Тем не менее стоит отметить, что ограничения, накладываемые данной моделью на характеристики управляющего, являются достаточно жесткими, что значительно ограничивает ее применение.

Иная мера была предложена Хенрикссоном и Мертоном, которые полагали, что менеджер-таймер в каждый момент времени пытается угадать, будет ли на следующем шаге избыточная доходность рыночного портфеля положительной или нет. При этом если он ожидает, что события начнут развиваться по первому варианту, то будет стремиться увеличить бету портфеля; если по второму - то уменьшить (Henriksson and Merton, 1981, Henriksson, 1991). То есть, в отличие от модели Трейнора и Мазуи, данный подход допускает резкое изменение беты, но накладывает более жесткие ограничения на реакцию менеджера. В общем виде доходность фонда может быть пописана следующим образом:

$$
\begin{aligned}
& r_{p, t+1}=\alpha_{p}+\beta_{p} r_{m, t+1}+\gamma_{p}\left(r_{m, t+1}\right)^{+}+e_{p, t+1}, \\
& \text { где }\left(r_{m, t+1}\right)^{+}=\max \left[0 ; r_{m, t+1}\right] . \text { Согласно интерпретации Хенрикссона и Мертона данное }
\end{aligned}
$$
выражение характеризует объем премии по опциону на доходность рыночного портфеля с ценой исполнения по безрисковой доходности. Более того, равно как и в безусловном варианте предыдущей модели, $\gamma_{p}$ будет отражать способности управляющего к таймингу.

Условная версия данной меры, предложенная Ферсоном и Шадтом, предполагает, что менеджер старается угадать отклонение рыночной доходности в будущем периоде от ее условного по множеству общедоступной информации среднего значения (Ferson and Schadt, 1996). При этом в зависимости от того, ожидает он бычий или медвежий рынок в будущем, соответственно будет определяться бета:

$$
\begin{aligned}
& \beta_{B U L L}=\beta_{0 B U L L}+B_{B U L L}^{\prime} z_{t} \\
& \beta_{\text {BEAR }}=\beta_{0 B E A R}+B_{B E A R}^{\prime} z_{t} .
\end{aligned}
$$

В итоге условная версия меры Хенрикссона - Мертона может быть записана в виде:

$$
r_{p, t+1}=\alpha_{p}+\beta_{0 B E A R} r_{m, t+1}+B_{B E A R}^{\prime}\left[z_{t} r_{m, t+1}\right]+\gamma_{p}\left(r_{m, t+1}\right)^{+}+\Delta^{\prime}\left[z_{t} r_{m, t+1}\right]^{+}+e_{p, t+1},
$$




$$
\begin{aligned}
& \text { где }\left(r_{m, t+1}\right)^{+}=\left(r_{m, t+1}\right) * I\left[\left\{r_{m, t+1}-E\left(r_{m, t+1} \mid Z_{t}\right)\right\}>0\right] \\
& \gamma_{p}=\beta_{0 \text { ВULL }}-\beta_{0 \text { BEAR }} \\
& \Delta^{\prime}=B_{\text {BULL }}^{\prime}-B_{B E A R}^{\prime} .
\end{aligned}
$$

В данном случае $I$ представляет собой бинарную функцию, идентифицирующую прогноз доходности в положительной зоне. Способности к таймингу при этом будут характеризоваться величиной $\gamma_{p}+\Delta^{\prime} z_{t}$, отражающей превышение условной беты над безусловной при высокой рыночной доходности, и наоборот. Тем не менее стоит отметить, что условные модификации стандартных мер также имеют определенные недостатки, в частности, они не позволяют выявить, какие из факторов информационного множества оказывают наибольшее влияние на значение оцениваемых параметров (Muravyev, 2006).

\section{Заключение}

Мощность и состоятельность атрибуционных мер определяется двумя основными аспектами: насколько можно разделить способности к выбору времени входа на рынок от способностей к выбору актива; какова разница между частотой фактического совершения операций и частотой выборки. В действительности менеджер может увеличивать получаемую доходность как за счет выбора актива, так и за счет выбора времени инвестирования. Для того чтобы не принимать одно за другое, предполагается справедливость двух допущений: информация менеджера для выбора актива независима от информации для выбора времени входа в позицию и портфель не содержит деривативов. При подобных предпосылках каждая бумага будет иметь малую долю в диверсифицируемом портфеле и не будет влиять на рынок.

Таким образом, на данный момент времени под атрибуционным анализом эффективности, как правило, понимается определение добавленной стоимости активного управления и ее последующее разделение на составляющие, сформированные за счет способностей менеджеров к выбору активов и выбору времени инвестирования. Подобный подход позволяет получить более точные оценки навыков, чем однофакторные меры в условиях нестационарности риска портфеля, характерной для реальных ситуаций.

\section{Список литературы}

1. Управление портфелем / пер. с английского С.З. Шукунда. ГИФА. 2006.

2. Abrevaya, J., Jiang, W. (2001), Pairwise-slopes statistics for testing curvature. University of Chicago Graduate School of Business and Columbia Business School: Working paper.

3. Admati, A.R., Bhattacharya, S., Ross, S.A., and P. Pfleiderer (1986), On timing and selectivity, Journal of Finance, 41 (1986) 715-730.

4. Bildersee, J.S. (1975), The association between a market determined measure of risk and other measures of risk, Accounting Review, 50 (1975) 81-98.

5. Breen, W., Glosten, L., Jagannathan, R. (1989). Economic significance of predictable variations in stock index returns, Journal of Finance 44 (1989) 1177- 1189.

6. Brinson et al. (1986), Determinants of Portfolio Performance, Financial Analysts Journal, July/August (1986).

7. Carhart, M. M. (1997), On persistence in mutual fund performance. The Journal of Finance, 52(1) (1997) 57-82.

8. Christopherson, J. A., Wayne E. F., and Glassman, D. A. (1998), Conditioning manager alphas on economic information: Another look at the persistence of performance, Review of Financial Studies 11 (1998) 111-142.

9. Cuthbertson, K., Nitzsche, D. (2004). Quantitative financial economics. Stocks, bonds and foreign exchange. John Wiley and sons, Ltd. 
10. Fama, E. and French, K. (2009), Luck versus skill in the Cross Section of Mutual Fund Returns, journal of Finance, 31 (2009).

11. Fama, E. (1972), Components of investment performance, Journal of Finance, 2(27) (1972) 551-567.

12. Fama, E., and French, K. (1993), Common Risk Factors in the Returns on Stocks and Bonds, Journal of Financial Economics, 33 (1993) 3-56.

13. Ferson, W.E., and Schadt, R.W. (1996), Measuring fund strategy and performance in changing economic conditions, The Journal of Finance, 51(2) (1996) 425-461.

14. Foster, G. (1986), Financial Statement Analysis, Prentice Hall, Englewood Cliffs.

15. Muravyev, D., (2006), Performance of Russian mutual funds, NES Working Paper.

16. Glassman, D.A., and Riddick, L.A. (2003), Market timing by global fund managers, Working Paper SSRN.

17. Goetzmann, W., Ingersoll, Jr., J., and Ivkovich, Z. (2000), Monthly measurement of daily timers. Journal of Financial and Quantitative Analysis, 35 (2000) 257-290.

18. Graham, J., Harvey, C., 1996. Market timing ability and volatility implied in investment newsletters' asset allocation recommendations, Journal of Financial Economics 42, 397421.

19. Grinblatt, M., and Titman, S. (1989), Portfolio performance evaluation: old issues and new insights, Review of Financial Studies, 2 (1989) 393-421.

20. Henriksson, R., and Merton, R. (1981), On market timing and investment performance: II. Statistical procedures for evaluating forecasting skills, Journal of Business, 54 (1981) 513533.

21. Henriksson, R.D. (1984), Market timing and mutual fund performance: An empirical investigation, Journal of Business, 57 (1984) 73-96.

22. Hochman, S. (1983), The beta coefficient: an instrumental variable approach, Research in Finance, 4 (1983) 392-407.

23. Jagannathan, R., and Korajczyk, R. (1986), Assessing the market timing performance of managed portfolios. Journal of Business, 59 (1986) 217-235.

24. Jensen, M. (1969), Risk, the Pricing of Capital Assets, and the Evaluation of Investment Performance, Journal of Business, 42 (1969) 167-247.

25. Jensen, M. (1972b), Optimal utilizationof market forecasts and the evaluation of investment performance. Mathematical Methods of Investment and Finance.

26. Jensen, M.C. (1968), The performance of mutual funds in the period 1945-1964, Journal of Finance, 23(2) (1968) 389-416.

27. Jiang, W. (2003), A nonparametric test of market timing. Journal of Empirical Finance, 10 (2003) 399-425.

28. Ke, D. (2006), On market timing ability: evidence from balanced funds. Eastern Kentucky University Press.

29. Kon, St. and Jen F.C. (1978), Estimation of time-varying systematic risk and performance for mutual fund portfolios: An application of switching regression, Journal of Finance, 33 (1978) 457-476.

30. Mamaysky, H., Spiegel, M., and Zhang, H. (2002), Estimating the Dynamics of Mutual Fund Alphas and Betas. Yale School of Management, ICF Working.

31. Mandelker, G.N., and Rhee, S.G. (1984), The impact of the degrees of operating and financial leverage on systematic risk of common stock, Journal of Financial and Quantitative Analysis, 19 (1984) 45-57.

32. Muravyev, D. (2006), Performance of Russian mutual funds, NES Working Paper Forthcoming.

33. Palmiter, A.R., and Taha, A.E. (2008), Mutual Fund Investors: Divergent Profiles, Working Papers.

34. Sewell, M. (2007), Fund Performance.

35. Sharpe, W. (1991), The arithmetic of active management, Financial Analysts, 47 (1991) 7- 
9.

36. Treynor, J., and Black, F. (1973), How to Use Security Analysis to Improve Portfolio Selection, Journal of Business, 46 (1973) 66-86.

37. Treynor, J., and Mazuy, K. (1966), Can mutual funds outguess the market? Harvard Business Review, 44 (1996) 131-136. 